Se]fe, c., and J. Ei]o]a. 1988. The tie that binds: Building discourse communities and group cohesion through computer-based conferences. Collegiate Microcomputer 64:339-348.

Smith, P., and M. Kelly. ]987. Distance Education and the Mainstream. London: Croom Helm.

Spiro, R. J., P. J. Feltovich, M. J. Jacobson, and R. L. Coulson. 1992. Cognitive flexibility, constructivism, and hypertext: Random access instruction for advanced knowledge acquisition in ill-structured domains. In Constructivism and the Technology of Instruction, eds. T. M. Duffy and D. H. Jonassen. Hillsdale, NJ: Lawrence Erlbaum.

Turoff, M. 1995. Designing a Virtual Classroom. Paper presented at the International Conference on Computer Assisted Instruction. URL: http://www.njit.edu/Department/cccc/vc/Papers/design.htmi.

Winklemans, T. 1988. Educational computer conferencing: An application of analysis methodologies to a structured small group activity. Unpublished Master's Thesis, University of Toronto, Toronto, Canada.

Wiggins, G. 1993. Assessment: Authenticity, context, and validity. Phi Delta Kappan 75:200-214.

Wildman, T., and J. Burton. 198 I. Integrating learning theory with instructional design. Journal o.lInstructional Development (4):5-14.

Winn, W. 1975. An open system model of learning. AV Communication Review 23:5-33.

Winn, W. 1990. Some implications of cognitive theory for instructional design. Instructional Science 19:53-69.

\section{Transactional Issues in Distance Education: The Impact of Design in Audioteleconferencing}

Terry D. Anderson and D. Randy Garrison

Abstract

\begin{abstract}
This paper reports the results of a study of student perceptions of learning via audioconferencing in university-level courses delivered at a distance. Field observations, interviews, and a focus group were used to triangulate and deepen knowledge obtained from an initial mail survey. The results of the investigation revealed that the audioteleconferences were being used under two quite different instructional designs, which were associated with significant differences in students' perceptions of the opportunity to engage in critical thinking skills, creation of a community of inquiry, and the value of various components of the distance education system. The study documents students' perceptions and outlines ways in which these sessions can be designed to induce maximum learning opportunities.
\end{abstract}

Introduction

Recent research on learning in complex environments (Suchman 1991; Cervero 1990; Rogoff 1990) has underscored the necessity of understanding the influence of the context in which learning takes place. Context is an important consideration in social-cognitive perspectives (Resnick, Levine, and Teas]ey 1991) and constructivist (Duffey and Jonassen 1992) views of learning. The socia] context of learning has been examined by researchers interested in cooperative learning (S]avin 1991; Johnson and Johnson 1979), situated cognition (Brown, Collins, and Duguid 1989), peer learning (Damon and Phelps 1989; Damon 1984), collaborative development of critical thinking skills (ThayerBacon 1993), and the development of communities of inquiry (Lipman 1991; Christensen 1991). A]though these studies of learning in a socia] context provide interesting clues to learning at a distance, they have focused on face-to-face instruction and interaction. A great deal of theoretica] and empirical research is necessary to understand and appropriately apply these educational theories and techniques in a distance context. 
The purpose of this study was to explore the influence of teacherstudent and student-student communication in terms of developing a community of inquiry and critical thinking ability. More specifically, instruments and research questions were used to explore students' perceptions of learning in an audioteleconferencing context.

\section{Literature Review}

This study is grounded in the practice of distance education, especially in that type of distance education that makes use of interactive telecommunications technology. The review of the literature that follows centers I) on the effect of telecommunications technologies on the defining characteristics of distance education and 2) on the effect of instructional design on the perceptions of learning in mediated contexts.

Distance Education and Interaction. Traditionally, distance education has been characterized as an individual form of learning (Holmberg 1986) lacking opportunities for socially shared learning. The emphasis on individual students studying alone at their own pace creates unique challenges to educators attempting to assist learners in cognitive development, especially that associated with social cognition. Keegan (1986) argues that noninteractive distance education must attempt to compensate for the following characteristics:

- no heard language

- absence of non-language communication

- absence of feed-back processes student-to-teacher

- absence of feed-back processes teacher-to-student

- delayed reinforcement

- absence of student-to-student communication

- change in role of non-cognitive learning processes (pp. 121-22)

According to Keegan, lack of compensation in any of these area results in a tendency for students to drop out, difficulty in achieving quality of learning, and a loss of status of learning. Efforts have been made to compensate by providing a variety of additional student support services (Amundsen and Bernard 1989), specialized writing styles (Holmberg 1989), and feedback mechanisms (Howard 1987).

The application of audio communications technology to distance education provides the opportunity to significantly alter six of Keegan's seven defining characteristics. Only Keegan's second characteristic, "abscnce of non-language interaction" (i.e., visual images), defines both traditional distance education and that supported via interactive audioteleconference. The defining characteristic of audioteleconferenceenhanced distance education is a substantial increase in human interaction; this increase has the potential to markedly change the nature, practice, and context of the distance education experience..

Moore (1989) describes three types of interaction relatIve to dIstance education: learner-content interaction, learner-teacher interaction, and learner-learner interaction. Learner-content interaction is the basis for, and defining characteristic of, all types of education. Included in this concept is the internal, didactic interaction between I arners. and oneway media, including correspondence text, and electro lc media such as broadcast television, interactive videodisc, and audio tapes (Moore 1989).

Learner-teacher interaction is regarded as essential by most distance and face-to-face educators. The goal of such interaction is to stimulate, motivate and facilitate educational activities and use of learning strategies. Co;respondence education provides significant $\operatorname{adv} \sim \mathrm{nt} \sim \mathrm{g} \sim \mathrm{s}$ in this area since interactions between student and instructor are II1dlvldual and not impaired by the necessity of meeting group needs. The provisio of feedback and the testing of new knowledge are the most valuable features of student-instructor interaction (Moore 1989). Do students perceive those interactions between teacher and student that are n )tprivate-that take place within the social context of the audioteleconference-as a valuable contribution to their learning or do they see them as a waste of precious individual learning time? Additionally, is the direct provision of feedback from teacher to student th most. valued component of the educational experience or is the creation of. new knowledge by the social learning group an equally valued part of the educational teleconference?

The etTect of learner-learner interaction has been little studied within a distance education context. Only those types of distance delivery that support group-based-as opposed to individual-study. ha e begu 1 to address this form of interaction. Learner-learner interaction IS nghtfully described by Moore as "a new dimension of distance education that will be a challenge to our thinking and practice in the 1990s:' (1989: 1(3). This study attempts to provide a first step in understandll1g the Importance of these interactions within an audioteleconferellce context 


\section{Instructional Design}

Despite warnings by Clark $(1983,1994)$ that the "media are mere vehicles that deliver instruction, but do not influence achievement any more than the truck that delivers our groceries changes our nutrition" (Clark 19 3, 445), educators have been searching for the "effect" of the medium on learning. Not surprisingly, the results of this search have been inconclusive (Kozma 1991,1994). Media can be used in many different ways to support and enhance learning. Additionally, the students using the media can adapt their learning behaviors and styles to accommodate the strengths and weaknesses of the media. Thus, to discover what learners or teachers like, dislike, or find effective or ineffective about any particular medium without consideration of the instructional design and other components of the learning context tells us very little. Draper et al. note that "what performs more or less well is not some material or medium (a lecture, a book, a computer program) but the whole learning and teaching episode" (1994, 7).

In order to ensure that the medium is not isolated from its context, this study has investigated the contexts in which the audioteleconferencing medium is used and seeks to uncover variations in instructional design or usage that influence students' perceptions of learning. The distance education field will advance when we can appreciate that the effect of the medium itself is only one factor in the entire teaching-learning context.

\section{Research Design}

The research questions and instruments in this study focused on the students' perceptions of learning during the audioteleconfcrence sessiems. Theories of adult education stress the capacity of adults to critically and collaboratively assess their learning experiences (Knowles 1984). Volet $(1991,322)$ notes that "a teaching-learning approach that students themselves recognize as effective and enjoyable is likely to produce better results and is more likely to be successful than one that encounters students' reticence, dislike or doubt about its effectiveness." Entwistle (1991) also emphasizes the importance of students' perceptions of the learning context as a crucial indicator of learning: "it is perceptions of the learning environment that influence how a student learns, not necessarily the context itself' (p. 202). Thus, investigating students' perceptions of the educational experience is an effective and valid measure of educational innovation.
This study examined the perceptions of learners enrolled in distance education courses that included scheduled, interactive sessions delivered via audioteleconferencing. The classes were delivered synchronously, usually in the evenings. The courses used audio-only, rather $t \sim a n$ aud.iographic, technology. Audioconferencing, a "low tech" but interactive medium, is the most cost-effective and, currently, the most common form of interactive distance delivery in Canada (Stammer 19 7).

The students in this study traveled to learning centers in their local communities that, on average, were over 150 kilometers from the major campus of the delivering university. Typically, two to four students attended together at a learning center, although $38 \%$ of the students attended classes in which they were alone at their learning center. The teacher was located at the site of the delivering university; in all but one case, no students were in the same room as the teacher. The twenty-four courses delivered by two Canadian universities represented the undergraduate, graduate, and credit diploma levels.

The study used a mixed-method design in which a quantitätive methodolon $_{b}$ y (a mail survey) was used in conjunction with qualitative methodologies (teleconferencing observations, semi-structure $d$ interviews, and a focus group). Data analysis proceeded sequentially: the survey data was analyzed prior to beginning the observa.tions and $\sim$ nterviews. The preliminary analysis of the survey data provided a basIs for the development of interview questions and analysis of the resulting qualitative data. This type of analysis is referred to as typol?gy development and is viewed as a particularly powerful means to Integrate the quantitative results-the "what"-with qualitative results-the "why" (Caracelli and Greene 1993).

The authors designed and pilot tested the survey instrument, which was mailed to all distance education students at University A $(11=133)$ and to one in three randomly selected distance education students from University $B(11=139)$. An overall return rate of $59 \%$ produced a sample of 160. The survey consisted of four demographic questions (age, gender, experience with teleconferencing, and number of stude ts at $\sim$ endi $\sim g$ at local sites), eight Likert-scale questions related to perception ot participation in a community of inquiry (Lipman 1991), twenty Likert-scale questions related to perception of opportunity to engage in critical thinking (Garrison 1991), and nine Likert-scale questions assessing students' perceptions of the effectiveness of the distance learning system components. The survey concluded with four open-ended questions related to the perceived effectiveness of the interactive components of the course. 
To complement the survey data, the researchers observed twelve c,lasses in three co munities. Because this was an exploratory investigatIOn, no predeter:nlOed, structured observation system or rating scale was used. However, 1Oterpersonal communication was the major focus of the obse vations: The investigators attempted to remain as inconspicuous as poss,lble du $1 \mathrm{Og}$ the observations, although the impact of an observer on t e 1OteractlOns, especially in very small classrooms, is impossible to discount.

Eighteen of the students attending teleconference courses were selected by invitation and interviewed. The semi-structured interviews were conducted on ,a schedule developed after analysis of the survey data. Iass observatIOns followed the same schedule. The length of the interviews anged from 25 to 45 minutes; transcripts were analyzed using the Atlas/tl (Muhr 1991) qualitative analysis program. Results of the initial analysis of the interviews and observations were mailed to the interview respon?ents., An audioteleconference focus group and individual telephone lOtervlews were used to confirm the findings.

\section{Results}

Demographic Characteristics oj'Leamers, The students in the survey sample were generally older adult students (47\% were 40 years old or older), The r latively high age of the subjects reflects the professional

ev lopment, 10 erests of these students, most of whom are mature professIOnals wlshlOg to expand their career potential. The students could be $b \sim s t$ describ d as mature, career-oriented adult learners who brought prevIOus educatl,on 1 , and vocational experiences to the learning context. There were no sl mhcant relationships between the survey items and the gende or age of the students, their previous experience with distance educ ltlOn, or the number of students attending class at the same remote locatIOn,

Desig 1 Models, As the investigation proceeded to the interview and obser atlOn phases, two quite different models of audioteleconferencina pra tlce became apparent. These two models differed on numerou social and organizational characteristics, and post-hoc analysis of the survey data showed significant differences in perceptions of learning between the two groups, The two models are labeled the Community of Learners (COL) model and the Independent Learning Support (ILS) model. The characteristics of these two models, illustrated by students' comments, are described in the following section.
The Community of Learners (COL) instructional design model had the following characteristics:

- frequent (usually weekly) audioteleconference meetings throughout a semester

- absence of a directed learning package, making successful completion of the course dependent on participation in the audioteleconferences

- emphasis on developing a stable, "virtual" classroom milieu in which students and teachers come to know each other through frequent audio interaction

- learning activities (e. g., structured discussion, student presentations, debates, and guest speakers) designed to bring out and develop existing skills and knowledge from within the group - course content, evaluation criteria, assignments, and text determined by the audioteleconference teacher, often in consultation with students

The emphasis on developing, extracting, and refining existing knowledge from the group, rather than on the teacher's transmission of knowledge to the students, is illustrated in student comments:

...what we want to do...is, in fact, take advantage of all the people that have the information and get them to bring their experiences out and not be just reading off all the information that they could read themselves in the book. So the idea is to try to put some of ourselves into it as well, but the real resource that the people don't have access to happens to be the opinions and thoughts of other people, so we have to incorporate that into it. I think that's essential for us. (COL student)

I have been learning to become more reflective and discerning of subject matter and of my own opinions and have been learning to accept the positions of others, because the teleconference has explored their understanding and consequently encouraged me to change or reconsider my own. (COL student)

The Independent Learning Support (ILS) instructional design model differs from the COL model in several respects:

- irregular and/or infrequent teleconference sessions (usually held once every two to four weeks) 
- optional attendance, resulting in instructor reluctance to deviate from prescribed content for uncertain numbers of students

- audioteleconferences viewed as an enhancement or adjunct to the learning package, which provides all activities, resources, and information necessary to successfully complete the course

- audioteleconferencing learning activities that focus on review of material from the package and on clarification of assignments and evaluation procedures rather than on new materials or issues

- course content and evaluation prescribed by the course designer and not open to negotiation or major changes

The ILS instructional design model used the teleconferences as opportunities for students to have immediate contact with other students and with the instructor concerning either course administration or Course content..A secondar fu ction was to provide a socially supported pacing mechamsm by settmg tlInes and deadlines by which course materials should be read and assignments completed.

The infrequency of teleconference sessions in this model limited opportunities for students to get to know other students either on-site or off-site. It was not unusual to observe classes in which students did not $\sim$ no,: each other's names or the names or numbers of students participat$\mathrm{mg}$ trom othe $\sim$ locations. One student noted that this information may even be unavallable to the teacher when she remarked that "very few of them [the teachers] will take attendance, so they don't really know who's out there."

he .learning activities using the ILS model consisted in large part of revlewmg chapters from the text or study guide, discussing assignments and tests, and answering specific student questions. There was little use of ?iscussion, debate, student presentation, guest speakers, or other ennchment activities. This format, with its emphasis on directive teaching and instrumental learning, seemed to meet the learning needs of many students, as illustrated by the following comments:

For the most part, what I find helpful at those [teleconferences] is discussion of papers that are coming up, answering questions about the content of them, and exams. (ILS student)

whe odd time we're asked to do something extra to help you learn a httle more or to help it stick a little more. I really hate doing them I really only want to do the textbook, the notes, whatever. (ILS student)
There was also a group of students who reported a strong sense of dissatisfaction with this model of instructional design. For these students, reviewing the material that had already been read without opportunity for application or further development represented a waste of their learning time:

...the stuff is already in the textbook anyways... and on the tapes. I just find them [the teleconferences I redundant. (ILS student)

Well, it's mostly answering questions from different students. I think I would enjoy it more if it was discussions I would like to get into a discussion with people from other communities and see what they think... and make it pertinent to what's going on in our times right now. (ILS student)

The differences between the COL and ILS learners were further illustrated by a post-hoc analysis of their responses to the community of inquiry, critical thinking, and learning resource items in the mail survey.

Community oj'lnquiry. Analysis of results using MAN OVA showed significant differences between the two groups (COL and ILS) of audioteleconference students (Wilks' criteria $F(8,139)=2.85, \mathrm{p}<. \mathrm{OI}$ ).

Table I presents the mean scores on each of the eight community-ofinquiry items. On all eight items the COL students perceived the audioteleconferences as supporting the development of a community of inquiry to a greater degree than did the ILS students. On the five items most associated with social interaction (opportunity for in-depth discussion, cooperative problem solving, opportunity for sharing, receiving feedback, and feeling of inclusion in the class), the COL students perceived significantly greater opportunities to share in a community of inquiry. It was only on the items related to reception of feedback, comments of other students, and opportunity for input into course materials that the differences between the two samples were not significant.

This analysis revealed that the COL students perceived the audioteleconference technology as supporting the development of a community of inquiry. This community functions without the benefit of the nonverbal communication channels used in face-to-face interaction, but it does operate, even when filtered through the teleconferencing medium. The ILS students' much lower perception of a similar community of inquiry illustrates that merely using the technology does not guarantee creation 


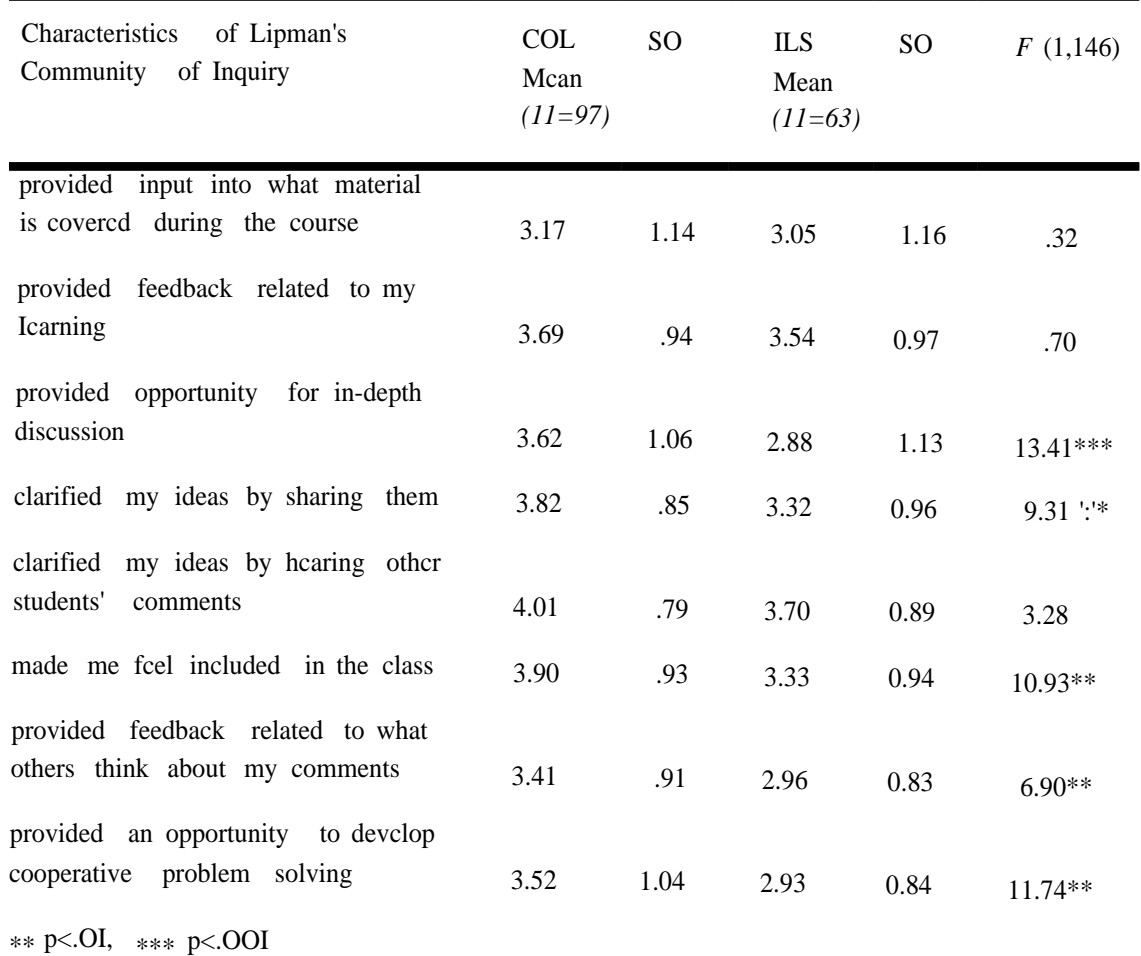

o '. c com unity of inquiry. We see in these results the strong influence of different 111structional designs on the perception and use of educational technologies.

Critical Thinking. To further explore the effect of design on the quality of learning, we assessed students' perceptions of opportunities to engage in critical thinking within the two models. The results of this analysis (Anderson and Garrison 1995) confirmed the significant difference in perception of learning opportunity between the two instructional design groups. MANOYA tests of difference between the two sample groups were significant [Wilks' criteria $F(5,133)$ $p<.001]$, and subsequent ANOYA tests showed significant differences $(p<.0 \mathrm{I})$ between mean scores on each of the five phases of the critical $t \sim$ inking cycle. In every case, the mean score of the COL sample was higher than that of the ILS sample, indicating greater perception of the opportunity to engage in critical thinking. Factor analysis of the critical thinking items provided general support for Garrison's (1991) model of critical thinking. However, in addition to the factors suggested from the model, a factor relating to the impact of formal assessment emerged, illustrating the pervasive influence of assessment on formal postsecondary education (Ramsden 1992).

Learning Resources. To assess the perceived efficacy of the learning resources and support components of the distance education system, survey subjects were asked to rate the effectiveness of a variety of learning resources and support services. Table 2 reports the means from the nine learning resources items for each sample. (A score of one indicated that the resource was very ineffective; a five indicated that the resource was a highly effective component of learning.)

\section{Table 2. Comparison of COL and ILS Samples on Learning Resources Variables}

\begin{tabular}{llllll} 
Learning Resource or Support & $\begin{array}{l}\text { COL } \\
\text { Mean }\end{array}$ & SO & $\begin{array}{l}\text { ILS } \\
\text { Mean }\end{array}$ & SO & F(L 138) \\
\hline Text & 4.11 & .46 & 4.30 & .50 & $5.28^{*}$ \\
Audiotcleconference & 4.14 & .70 & 3.55 & .79 & $15.17^{* * *}$ \\
Learning package & 3.91 & .71 & 4.23 & .86 & $6.95^{* *}$ \\
Other students on-site & 4.08 & .78 & 3.60 & .89 & $19.40^{* * *}$ \\
Teacher & 4.24 & .89 & 3.77 & 1.02 & $8.89^{* *}$ \\
Other students, off-site & 3.66 & .77 & 3.29 & .92 & $7.00^{* *}$ \\
Others, not enrolled in the course & 3.37 & .86 & 3.45 & .86 & 0.26 \\
Library staff and resources & 3.39 & 1.09 & 3.53 & .86 & 0.68 \\
$\begin{array}{l}\text { Support staff employed by } \\
\text { the University }\end{array}$ & 3.75 & 1.02 & 3.42 & .88 & $4.81^{*}$
\end{tabular}

$* \mathrm{p}<.05 ; \quad * * \mathrm{p}<. \mathrm{OI} ; \quad * * * \mathrm{p}<. \mathrm{OOI}$

MANOYA tests conducted on the learning resources variables showed no significant differences between the groups based upon gender, age, experience with teleconferencing, or number of students at the local 
teleconference site. MANOVA tests, using model of instructional design as the independent variable, were highly significant [Wilks' criteria = $.7115, F(9,130)=5.73, p<.001]$. The results of univariate analysis of variance are displayed in Table 2 .

Seven of the education resource items showed significant differences between the two samples. The two items related to independent studytext and learning package-produced significantly higher perceived effectiveness rates among the ILS students. On the four items associated with social influences on learning, the COL students had significantly higher scores. The COL sample also had a significantly higher score on the perceived effectiveness of university support staff. There were no significant differences between the two samples on the items related to others who are not in the course or to library staff. These results indicate that the perception of effectiveness of learning resources is very different between the two sample groups.

The COL students perceived greater opportunity to engage in socially shared learning and, further, placed a higher value on these experiences. ILS students, on the other hand, perceived significantly greater value in the independent study resources, suggesting that their experience of learning in these courses was largely an independent process. The social components of learning were valued much less by the ILS students. Although the finding that students valued the type of educational experiences with which they have had most experience is not surprising, it does confirm the different perceptions of learning and the different value placed on the associated tools of learning between these two sample groups. Interestingly, the learning resource with the highest perceived value in the ILS sample, and the second highest in the COL sample, was the print-based textbook. There was high perceived value in print-based materials that could be used individually, even when the teleconferences provided significant opportunities for learning in a social context. It is also not surprising to note that students perceive the learning value of students on-site with them to be higher than the value of students located at other sites.

The findings from the learning resources data illustrate that students using different models of teleconference-based distance delivery place different value on the educational resources provided by the educational institution. These results also illustrate the complexity of building in student-support mechanisms, a process influenced by the instruction and learning activities encapsulated in the instructional design that defines the course. Different instructional designs and desired learning outcomes will invite different solutions to achieve the optimal mix of studentsupport services.

\section{Discussion}

The data from both the survey and the qualitative tools indicate that these models represent two distinct forms of audioteleconferencing. Students in courses based on both models of instructional design perceived the teleconferencing context as supportive of Lipman's (1991) "community of inquiry." However, the extent of this perception of community was associated with the instructional design which underlay the intended function and learning activities engaged in during the teleconference. Secondly, students generally did perceive the teleconferencing context as providing an opportunity to experience and develop critical thinking skills. Again, however, the extent of this perception was linked to the instructional model upon which the teleconference is designed.

The audioteleconference session, in both ILS and COL instructional design models, dictates that students be situated at one of a number of given sites at a given time. The group teleconference encourages students to match the pace of their learning activities to the progress of the class. The vast majority of responses to the open-ended and interview questions revealed that most students $(83 \%)$ in this study appreciated the pacing that accompanies a regularly scheduled teleconference course. The busy lifestyle of many adult learners makes it difficult to allocate blocks of time for reading and studying (Wilkinson and Sherman 1990). The scheduled teleconference provides a time when students can leave their home or business and devote time exclusively to the learning process. Both ILS and COL students regularly mentioned the value of the teleconference in establishing goals and timelines by which progress through the course was regulated. Many ILS courses have long reading lists and independent learning activities that demand considerable selfmotivation for successful completion. The teleconference provides a regular incentive to keep up with course work. A small minority of students reported that the time and place restrictions imposed by the teleconferences limited their ability to participate in the course. As in any educational delivery system, trade-offs must be made between accessibility and the benefits of paced and socially shared learning. The evidence from this investigation shows that most of the students enrolled in these courses were willing to accept the loss of some independence in exchange for the benefits of interactive and paced learning. 
The finding that independence of time and place is not a crucial concern for the majority of distance education students in this study has important ramifications for the development and practice of distance education. If the samples from this study are in any way representative of the majority of distance education students, then a fundamental premise of distance education as defined by some theorists (Holmberg 19 6; Keegan 19 6) is open to question. Many distance educators have refused to incorporate, or have relegated to "optional status," any interactions that restrict student access in terms of time or place. This ideological commitment to independent study denies interactive educational opportunities and choices to students and teachers.

Telecommunications technologies provide opportunities for learning at a distance which may be independent of or dependent on time, place, or both (e.g., computer-mediated communications is independent of both; audio and video teleconferencing are both time and place dependent). It is presumptuous of educators to assume that total independence of time and location is the most desirable or the most effective method of delivering quality distance education. This study docs not prove that sustained interaction between and among students and teachers is required for all types of learning to occur. It docs suggest that students' perceptions of the quality and value of their learning was significantly enhanced when meaningful opportunities for mediated interaction were provided.

The presence and participation of peers had significant effects on learning in the audioteleconference context. Socially shared learning took place among participants in three ways: among students at the same site (on-site), among students at different sites (off-site), and between students and teacher. Students perceived that interaction with other students at their site is important and can help them successfully complete the course by providing a support group: a "measuring stick" to evaluate individual performance and a "sounding board" for complaints and frustrations. The local group may also enhance learning by providing a forum to validate new knowledge gained during the course. Considerable evidence from both the survey and the interviews suggests that other on-site learners provided a uniquely informal learning support system. The effective use of on-site discussion-"sidetalk"-during the teleconference allowed students to affirm or dispute information provided by others without interrupting the rest of the teleconference class. The ongoing development of the learning community on site can facilitate a strong sense of group endeavor and support. Examples of this support included students working on class projects together and arranging to meet for work or study sessions outside the scheduled class time.

All students interviewed in this study reported that it was more difficult to develop and maintain student support groups with students at other sites (off-site peers). To support socially shared learning across geographic distances, students must be given regular opportunities to interact with students at other sites. Knowing and using the names of other students and using techniques to stimulate inter-site discussion facilitated socially shared learning with off-site peers. The instructional technique of creating small discussion groups by linking sites into "subconferences" seemed to be an effective way to develop these cross-site communities. Students studying by themselves at a site reported having greater difficulty feeling part of the class and often experienced problems participating in discussions with sites that included many students. Although their opportunities to participate were limited to communication through the teleconferencing medium, students alone at a site still valued the social interaction at a distance.

The success of the teleconferences was dependent on the teachers' ability to provide learning activities appropriate to students' needs, to manage the discussion, and to provide information of value to the students. The two instructional design models served different purposes and placed different emphases on the appropriate function of the teacher in a successful teleconference. The COL teacher was free to adopt many of the teaching strategies and activities used in the face-to-face classroom. Once a level of technical proficiency was achieved by participants, the teacher could lecture, initiate class discussions, moderate student presentations, or facilitate more experimental activities such as on-line debates, guest audio speakers, or panel discussions. The primary role of the teacher was to assist in the creation of knowledge through facilitation of interaction and critical discourse.

The ILS teacher faced a more daunting task when preparing for a teleconference. During the two-to-four week period between class sessions, the students were expected to read considerable amounts of material from the text and study guide. In addition, any assigned course work was either due or in the process of being returned to students. Finally, the teacher could never be sure how many sites would participate or how many students would attend the teleconference. Throughout the course, the ILS teacher had only minimal personal contact with most students and knew few students by either voice or name. As a result, many ILS teachers conducted teleconferences in a didactic manner, focusing on 
review and explanation of material in the text and learning package. Despite the didactic nature of the exchange, ILS teachers were careful to provide opportunities for students to ask questions or clarify difficulties with subject matter or administrative concerns. The role of the teacher was primarily that of a reactive information resource.

\section{Conclusion}

This study reveals the importance of human interaction as perceived by students registered in distance education courses delivered via interactive audioteleconference. The instructional design upon which the interactive sessions were planned and orchestrated significantly influenced students' perception of this learning. Merely acquiring and using the technology, without regard to the development of opportunity for regular and sustained interaction between and among teacher and learners, provided no guarantee that a critical community of learners would result. If critical thinking is a desired learning outcome, then learning activities that capitalize on the interactive potential of the medium must be planned and developed.

The results of this study emphasize the profound effects of instructional design on students' perception of learning. This study, in which the same technology was used to accomplish very different educational purposes, supports Clark's (1983) claim for the influence of design over medium. The two approaches to design identified in this study might be characterized as a "deterministic, highly specified and reproducible set of procedures [compared] to an enterprise that is dynamic, adaptable and suited more to entering the 'mental life ' of the learner" (Shale and Garrison 1994). The challenge is to match instructional design to instructional purpose. Designers of distance education programming need to be cog nizant of design approaches and of the educational goals of the program. The findings of this study suggest that different designs produce different outcomes. Unlike the typically unfruitful search for the general learning effect of any particular medium, studies that focus on the influence of instructional design on learning outcomes will be a productive focus for future research in distance education.

\section{References}

Amundsen, S., and R. Bernard. 1989. Institutional support for peer contact in distance education: An empirical investigation. Distance Education 100 ):7-27.

Anderson, T., and D. R. Garrison. 1995. Critical thinking in distance education: Developing critical communities in an audio teleconference context. Higher Education 29:83-99.

Brown, J. S., A. Collins, and P. Duguid. 1989. Situated cognition and the culture of learning. Educational Researcher 180 ):32-42.

Caracelli, V. J., and J. Greene. 1993. Data analysis strategies for mixedmethod evaluation designs. Educational, Evaluation and Policy Analysis 15(2): 195-207.

Cervero, R. 1990. A model of professionals as learners. In Visions for the Future of Continuing Professional Education, eds. R. Cervero and J. Azzaretto, 161-182. Athens, GA: University of Georgia.

Christensen, R. 1991. Premises and practices of discussion teaching. In Education for Judgment, R. Christensen, eds. D. Garvin and A. Sweet, 15-26. Boston: Harvard Business School Press.

Clark, R. E. 1983. Reconsidering research on learning from media. Review of Educational Research 53:445-460.

Clark, R. E. 1994. Media will never influence learning. Educational Technology Research and Development 42(2):21-29.

Damon, W. 1984. Peer interaction: The untapped potential. Journal of Applied Developmental Psychology 5:331-343.

Damon, W., and E. Phelps. 1989. Critical distinctions among three approaches to peer interaction. International Journal ()f Education Research 13:9-19.

Draper, S. W., M. Brown, E. Edgerton, F. Henderson, E. McAteer, E. Smith, and H. Watt. 1994. Ohserving and measuring the pClformance of educational technology. Glasgow UK: TILT Group, University of Glasgow.

Duffey, T., and D. Jonassen. 1992. Constructivism and the Technology ()f Instruction. Hillside, NJ: Lawrence Erlbaum.

Entwistle, N. 1991. Approaches to learning and perceptions of the learning environment. Higher Education 22:201-204.

Garrison, D. R. 1991. Critical thinking and adult education: A conceptual model for developing critical thinking in adult learners. International Journal of Lifelong Education 10(4):287-303. 
Holmberg. B. 1986. Growth and Structure of Distance Education. London: Croom Helm.

Holmberg, B. 1989. Status and Trends of Distance Education. London: Kogan.

Howard, D. C. 1987. Designing learner feedback in distance education. The American Journal qfDistance Education 1(3):24-40.

Johnson, R., and D. Johnson. 1979. Conflict in the classroom-Controversy and learning. Review qf'Education Research 49:51-70.

Keegan, D. 1986. The Foundations of Distance Education. London: Croom Helm.

Kn?wles, M. S. and ssociates, eds. 1984. Andragogy in Action: ApplyIng Modern Principles of Adult Learning. San Francisco: Jossey-Bass.

Kozma, R. 1991. Learning with media. Review of Educational Research 61 (2): 179-211.

Kozma, R. B. 1994. Will media influence learning? Reframing the debate. Educational Technology Research and Development 42(2):7-19.

Lipma , M. 1991. Thinking in Education. Cambridge: Cambridge University Press.

Moo e, M. 1989. Th.ree types of interaction. In Readings in PrinCljJles of Distance EducatIOn, ed. M. Moore, 100-105. University Park, PA: The American Center for the Study of Distance Education, The Pennsylvania State University.

Muhr, T. 1991. Atlas/ti: A prototype for the support of text interpretation. Qualitative Sociology 14(4):349-371.

Ramsden, p. 1992. Learning to Teach in Higher Education. London: Routledge.

Resnick, L., 1. Levine, and S. Teasley, eds. 1991. Perspectives on Socially Sh r d Cognition. Washington: American Psychological AssocIatIOn.

Ro?off, B. 1990. Apprenticeship in Thinking: Cognitive Developments In Social Context. New York: Oxford University Press.

Shale, D., and D. R. Garrison. 1994. Instructional design in distance education. Paper presented at the Annual Conference of the Canadian Association for Distance Education, Vancouver.

Slavin, R. 1991. Cooperative Learning TheOlY. Research and Practice. Englewood Cliffs, NJ: Prentice Hall.
Stammer, A. 1987. Communications technology and distance learning in Canada. Ottawa: Department of Communications, Government of Canada.

Suchman, L. A. 1987. Plans and Situated Action. New York: Cambridge.

Thayer-Bacon, B. J. 1993. Caring and its relationship to critical thinking. Educational Theory 43:323-340.

Valet, S. E. 1991. Modeling and coaching of relevant metacognitive strategies for enhancing university students' learning. Learning and Instruction 1:309-336.

Wilkinson, T. W., and T. M. Sherman. 1990. Perceptions and actions of distance educators on academic procrastination. The American Journal of Distance Education 4(3):47-56.

\section{Acknowledgments}

The authors wish to acknowledge the support of the Northern Health Human Resources Research Unit at Lakehead University in Thunder Bay, Ontario for financial and logistical support provided during this study.

Collference Announcement

VI International Conference on Technology and Distance Education "SHARING EXPERIENCES AROUND THE WORLD"

October 24-27, 1995
San Jose, Costa Rica
Sponsored by
Nova Southeastern University and Universidad Estatal a Distancia
For more information, contact

